\title{
,Aufbewahrungssysteme'. Anordnungen des Wissens im Literaturarchiv
}

\author{
die frage des sammelns ist gekommen. \\ das ansammeln ist darob noch vielfacher und geschachtelter.
}

(Schwab 1999, 94)

\section{1 „... ein paar Kisten in den Regalen“}

In Norbert Gstreins Roman In der freien Welt (2016) kommt eine Archivarin zu Wort, die für das Literaturarchiv der Nationalbibliothek in Wien tätig ist und auf die Anlieferung von „Kartons und Kartons von Papieren eines schon zu Lebzeiten mehr toten als lebenden Schriftstellers“ (Gstrein 2016, 71), durchaus einer österreichischen Beamtentradition folgend, mit häufigem Krankenstand reagiert. Die Archivarin erklärt ihr unglückliches Bewusstsein damit, dass „das bisschen Unsterblichkeit im Archiv“ sich in „ein paar Kisten in den Regalen“ materialisieren würde, in die ,womöglich nie wieder jemand hineinschaut[] und die dort verstauben“ (Gstrein 2016, 72).

Materialität und Nachruhm sind hier eng mit der Gegenstandsbestimmung der Kartons verknüpft, in denen sich das zukünftige Werk potenziell materialisiert und lokalisiert, also den Archivboxen, um den Terminus technicus zu gebrauchen. Dieser das Werk speichernde Garant für den (ungewissen) Nachruhm rückt dieses spezifische, Aufbewahrungssystem' des Literaturarchivs in seiner paratextuellen, aber auch kontextuellen Materialität ins Zentrum, worunter jene Phänomene zu fassen sind, die eben nicht nur eine die handschriftlichen Entwürfe und Brouillons, verschiedene Textstufen in Manuskript oder Typoskript umfassende physische Komponente, sondern gleichzeitig die „Realitäten institutioneller Zwänge und Machtstrukturen“ (Benne 2015, 84) bezeichnen.

Dieses Medium bestimmt die Lage der Archivalien im Literaturarchiv und verdient deshalb eine genauere Beschreibung. Medien, so ein Diktum Friedrich Kittlers, veralten im Fünfjahresrhythmus, im Fall des durativen Mediums Schachtel verhält es sich jedoch anders. Parallel zu den kittlerschen Aufschreibesystemen soll in der Folge von einem Aufbewahrungssystem die Rede sein, welches insbesondere in Archiven zur Anwendung gelangt und dort über mannigfaltige normative Funktionen verfügt: Im Objekt Schachtel kreuzen sich zahlreiche Diskurse, die die Historizität und Epistemologie der Verpackung betreffen, ebenso Papier- 
und Technikgeschichte, aber auch Archivierungstechniken und die damit verbundene Logik der Faltung umfassen.

Im Sinne Kittlers kann dieses Objekt als Knotenpunkt eines Netzwerkes von „Techniken und Institutionen“ (Kittler 2003, 501) begriffen werden, welches „die elementaren Funktionen des Aufzeichnens und Darstellens, des Speicherns, Verarbeitens und Überlieferns von Wissen“ (Wolf 2015, 118) bündelt. Die folgenden Ausführungen schließen auch an jene Überlegungen Ulrike Vedders an, dass Dingen, vor allem jenen millionenfach Reproduzierten, eine „wissenschaftstheoretische und -historische Relevanz“ (Vedder 2012, 17) zukommt. Die Schachtel, so kann man mit Wolfgang Ernst postulieren, ist ein „,Ordnungszeichen/Operator[] des Mechanismus““ (Ernst 2003, 44), - des ,dokumentverarbeitenden Wesens“ des Archivs. Perspektiviert aus Jacques Derridas ,Archivologie“ ist die Schachtel innerhalb der Institution Archiv als materielle „Konsignationsmacht“ wirkungsmächtig, da sie zum einen die „,topologische ,Ortszuweisung‘ bzw. die ,Aufbewahrung an einem Ort [...]', zum anderen das ,archontische Prinzip des Archivs‘, nämlich das ,Versammeln der Zeichen““ (Derrida 1997, 13), verkörpert.

Wirft man einen Blick in die Ordnungssystematiken zeitgenössischer Literaturarchive, ist die Schachtel zunächst v. a. quantitativer Indikator, bezeichnet ihre Anzahl doch den Umfang des jeweiligen Vor- oder Nachlasses: „Auf die Bezeichnungen Nachlaß, Teilnachlaß, Splitternachlaß [...] folgt, wo bekannt, die Umfangsangabe in möglichst aussagekräftiger Form. Die Größe von Kartons, Schachteln u. ä. kann allerdings stark differieren“ (Hall und Renner 1992, V-VI). Mit Roland Barthes möchte man jedoch dieser pragmatischen Beschreibung im archivarischen Kontext entgegenhalten, dass die Archivbox als ein eminenter Agent der Täuschung fungiert:

So spielt die Schachtel Zeichen: als Hülle, Schirm, Maske steht sie für das, was sie verbirgt oder schützt und gleichwohl bezeichnet: sie tauscht und täuscht. Aber was sie da umschließt und bezeichnet, wird sehr lange auf später verschoben, als läge die Funktion des Pakets nicht in einem räumlichen Schutz, sondern in einem zeitlichen Aufschub. In die Umhüllung scheint die Arbeit des Zurechtmachens (des Tuns) investiert, aber gerade dadurch verliert der Gegenstand seine Existenz, er wird zur Täuschung. (Barthes 2012, 65)

Ob sie in dieser Funktion das komplexe ,Gefüge“, die „Skriptomasse“ (Raulff 2009, 228) Nachlass auch enthält oder ihn mit „archivarischen, denkmalpflegerischen oder kuratorischen Strategien“ (Plachta 2017, 34) überlagert, muss an anderer Stelle untersucht werden. Darüber hinaus markiert die Schachtel im modernen Literaturarchiv, was zum ,Werk‘ eines Autors zählt und was dem ,Außen` zuzurechnen ist. Denn Objekte, wie z. B., um zwei österreichische Beispiele zu bemühen, der Schlafrock Heimito von Doderers oder der Spazierstock Peter Handkes, wandern in die Vitrinen von Literaturausstellungen. Die „Gretchenfrage“ des Literaturar- 
chivs ist, nicht nur hinsichtlich von Kanonisierungsprozessen, immer auch eine des Ein- und Ausschlusses, des ,Enthaltens', denn was aus dem Archiv wieder „einmal in den Blick rücken wird, weiß letztlich keiner“ (Schenk 2008, 56).

Diese Zukünftigkeit der Archivalien bildet sich zunächst materialiter ab, so entsteht „da vorzüglich, wo Staub auf dem Papiere liegt, unabwendbar Moder, der da Papier nicht nur unscheinbar macht, die Schrift vertilgt, gepappte Bände löst, sondern auch das Papier zerstört" (Sinnhold 1842, 25). Diesen unausweichlichen physischen Zersetzungsprozess verzögert das Dispositiv ,Schachtel‘; der „Geschmack des Archivs“ (vgl. Farge 2011) wird konserviert und verschoben auf unbestimmte Zeit. Die Archivbox ist demnach ein wesentliches mikropolitisches Element zur Sicherung des kulturellen Erbes in der makropolitischen Institution des Archivs. Im ,Organismus ‘ Archiv sind die Boxen ein wesentlicher Faktor, der die endo- und exogenen Okklusionsprozesse des Papiers verlangsamt (vgl. SchüllerZwierlein 2014, 41). Die Art der „dauerhaften Aufbewahrung“ in „geschlossenen Boxen aus Karton“ wird empfohlen, da die Boxen „das Schriftgut sowohl vor Licht, Nässe und Staub, aber auch mechanischer Beeinträchtigung schützen“ (HammerLuza 2007, 69).

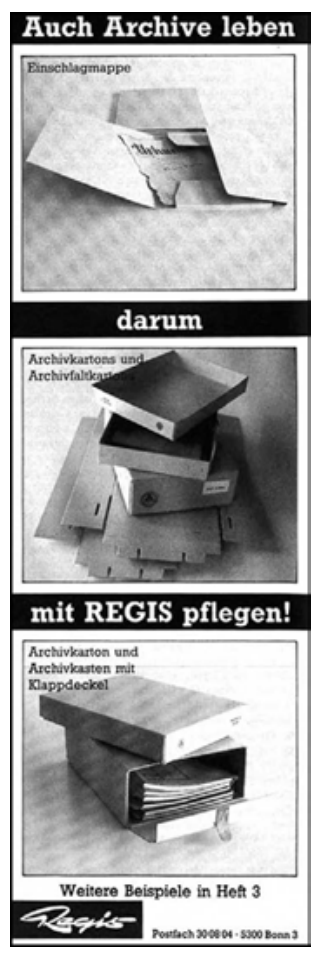

Abb. 1: Werbung der Firma Regis. 


\section{2 „Arca-logie“: Eine Wissenschaft von der Kiste}

Die Urheber von Wissen und Erkenntnis sind in Bezug auf die Institution Literaturarchiv ein „Ensemble aus menschlichen Agenten“, aber ebenso „Apparaturen und Artefakte[]“ (Günzel und Ebeling 2009, 14). In den folgenden Ausführungen sollen einerseits die Modalitäten der Aufbewahrung im Kontext der relativ jungen Institution Literaturarchiv in einen breiteren wissenschaftsgeschichtlichen Kontext gestellt werden und andererseits eine allgemeine Darstellung der Archivbox im Sinne einer „Behältertheorie“ des Archivs entworfen werden, wobei die Aussagen über „Behälter“ bezüglich der „Schachtel“ im weitesten Sinne eine begriffstheoretisch und -praktisch eher verschachtelte Angelegenheit sind. Die Archivterminologie führt, z. B. in der von Wolfgang Leesch herausgegebenen Archivkunde Adolf Brennekes im Index unter dem Lemma „Archivalienbehälter, -aufbewahrung“ folgende Verweise, Synonyme und Begriffe an: „Archa, Capsula, Cista, Armarium (ältere Bezeichnung für Archiv, Achivalienbehälter), Sacrarium, Scrinium (ältere Bezeichnung für Archiv, Archivalienbehälter), Segerer, Tresekammer, Domus chartarum, Turris chartularia“ (Brenneke 1953, 438).

Daraus wird ersichtlich, dass die heute eingesetzte Archivbox - in Form von Stülpdeckelbox, Frontklappenbox, Wickelbox oder Klappkassette (vgl. Glauert 2011, 60) - historische Vorläufer und eine eigene historische Figuration besitzt. Die Archivbox kann ihre Verwandtschaft zum Medium Schrank, aber auch zum Buch, dessen Analogie, wenn nicht Antithese sie gleichzeitig ist, nicht leugnen und hat eine eigene historische Ausdifferenzierung unterlaufen, die in Umrissen dargestellt werden soll. Das Lexikon des gesamten Buchwesens definiert den Begriff „Archiv- bzw. Schriftgutbehälter“ pragmatisch als „,der geordneten und geschützten Aufbewahrung des Schriftgutes“ dienend, die ,je nach Schriftguttyp und Benutzbarkeits- und Haltbarkeitsanforderungen unterschiedliche Formen“ (Leesch 2017) aufweist.

Bevor die Dinge in das kulturelle Gedächtnis Einzug halten können, müssen sie zunächst in eine topologische Reihenfolge gebracht werden, eben benutzbar und haltbar gemacht werden, denn „Wissen braucht Behälter“ (Heesen 2007, 91). Die Leistung, die ein Behälter erbringen muss, besteht nicht nur darin, die „Sammlung eine Sammlung“ bleiben zu lassen, also ihre Zerstreuung zu verhindern, sondern die einzelnen Stücke einer Sammlung „gleich einem Schild“ (Sommer 2002, 218) vor zerstörerischen Kräften zu schützen, wobei dem Behälter eine ein- und ausschließende Erhaltungsfunktion zukommt.

Im Kontext des modernen Literaturarchivs ist die Archivbox ein wesentlich konstitutives Element der Ordnung, die im Depot herrscht. Als ein modernes „Archivmöbel“ ist sie funktionell und schmucklos, in ihrem massenhaften Vorhandensein fast schon unauffällig, dennoch ist sie für eine Geschichte des 
Wissens und der Wissensanordnungen, die „gerade die Objekte und physischen Träger von Wissen miteinbeziehen möchte“ (Friedrich 2013, 72), von grundlegender Bedeutung, da den Objekten und Trägern, wie Schränken, Regalen oder Truhen als ordnungsstiftenden Sammlungsmöbeln, ebenfalls Aufmerksamkeit geschenkt werden sollte.

Was es mit der Archivschachtel als ,Aufbewahrungssystem' auf sich hat, lässt sich zunächst etymologisch zurückverfolgen, ist doch das Wort „Schacht“, ein „hohle[r] Raum“ und, so Krünitz’ Oekonomische Encyklopädie, mit dem Schrank verwandt. Als ein „,aus dünnen Seitenwänden bestehendes Behältniß, mit einem darüber passenden, genau anschließenden Deckel“ (Krünitz 1824, 481), ist sie von ihrer Materialität her aus all denjenigen Körpern gefertigt, die sich verarbeiten und verbiegen lassen.

Fachsprachlich wird nach Karton bzw. Pappe, also dem Werkstoff, und der Kartonage, dem eigentlichen Behälter, unterschieden. Im allgemeinen Verständnis beschränkt sich der Begriff Kartonage auf Behälter, bezeichnet jedoch auch sowohl die Bearbeitung von Pappe als auch das Endprodukt, zumeist Schachteln oder Kästen aus Pappe, die in Fabriken gefertigt wurden; in den älteren Manualen der Buchbinderei wird dieser Bereich als „Papparbeit“ bezeichnet (vgl. Poppe 1840). Diese Topologie des Faltens - im Sinne von Kanten produzieren -, hat Walter Seitter in seiner Physik der Medien eingehend beschrieben, die gerade Linie, welche quer über das Papier verläuft und dieses ,knickt‘, nähert die beiden Hälften des Papiers in der dritten Dimension einander an. Das Papier wird bei diesem Prozess nicht zerstört, aber ,er hinterläßt eine bleibende lineare Wunde oder Narbe“ (Seitter 2002, 200). Ein zentraler Begriff ist in diesem Zusammenhang die sogenannte „Zarge“, womit die „seitliche Einfassung eines räumlichen Gegenstandes bezeichnet“ (Grimm Bd. 31, 1854ff., 281) ist - die Grundbedeutung „Rand“, berührt sich etymologisch auch mit „Sarg“ -, während im Krünitz bereits darauf hingewiesen wird, dass dieser Begriff die Seitenwand einer Schachtel bezeichnet.

Direkt in die Geheimnisse des Archivs führt ein anderer etymologischer Faden: „Arcae“ werden Behältnisse genannt, die verschließbar sind, also Kisten und Truhen, Keller, Gefängniszellen. „Die im Archiv deponierten Akten der frühen Neuzeit“, so Cornelia Vismann, „gelten für lange Zeit als das schlechthin Weggeschlossene, Sekrete, Versiegelte. Und das, was sie umschließt, wird arca genannt“ (Vismann 2009, 102-103). Epistemologisch führt dies zum „arcanum“, also jenem „Mantel des Geheimnisses“, mit dem sich das Archiv umgibt, und der im rhetorischen Sinne als Figur oder Trope gelesen werden kann, der - im Sinne Derridas -, als ein „Bestimmungsempfänger“ gilt, „der die Kraft des [...] Geheimnisses bewahrt“ (Derrida 1997, 51). In einer ins Materialistische gewandten Leseart von Derridas „Archivkrankheit“ kann die Archivbox als eine „Konsignationsmacht“ 
beschrieben werden, sie bestimmt die „topologische ,Ortszuweisung bzw. die ,Aufbewahrung an einem Ort oder auf einem Träger‘, zum anderen versammelt sie als „archontisches Prinzip des Archivs“ (Derrida 1997, 13) die Zeichen.

Das Medium Archivbox ist also ein vielfacher Bezugspunkt innerhalb des Depots eines Archivs. Sie enthält schlussendlich, egal welcher Typologie von Nachlass die Papiere entsprechen (vgl. Meyer 2002, 52-58), stets dieselbe Ordnung nach den „Regeln zur Erschließung von Nachlässen und Autographen“ (RNA).

In Derridas Archivtheorie folgt aus der Erwähnung des Begriffs „arca“ (vgl. Derrida 1997, 48) keine Theorie des Archivs als Poetik des Raumes und keine Theorie des Archivs als Epistemologie des Containers (vgl. Vismann 2000, 103). Dieses spezifische „Aufbewahrungssystem“ im Kontext einer „Arca-logie“, wie Vismann sie vorschlägt, als einer Wissenschaft von der Kiste und ihren materiellen und dispositiven Voraussetzungen ihrer Anordnung, muss bisher als noch ungeschrieben gelten.

\section{3 ,Aufbewahrungssysteme‘: Von Ordnung und Anordnung}

Dass Wissensproduktion stets historischen Entwicklungen unterliegt und von diversen Faktoren abhängig ist, etwa der medialen Verfasstheit seiner Präsentation oder politischer und gesellschaftlicher Ordnungen und Entwicklungen, ist in der Forschung evident (vgl. Spoerhase et al. 2009). Die Ordnungen des Wissens sind umfangreich erforscht worden, u. a. unter dem Überbegriff des Sammelns, werden verschiedenste ,Aufbewahrungssysteme‘, rangierend von der Kunstkammer (vgl. Bredekamp 1993), dem Museum moderner Prägung (vgl. Heesen und Spary 2001) bis hin zum Zettelkasten (vgl. Gfrereis und Strittmatter 2013; Krawjeski 2017), Album (vgl. Kramer und Pelz 2013), aber auch Tisch (vgl. Pelz 2003) und dem Zeitungsausschnitt (vgl. Heesen 2006) umfassend behandelt. Mit dem Begriff „Anordnung“ soll ein epistemisches und technologisches Moment in die Diskussion eingebracht werden, denn mit der „Wendung von der Aufbewahrung zur Produktion des Wissens“, so Wolfgang Ernst (2002, 39), hat Foucault dem Archiv seine dokumentarische Passivität und konservierende Unschuld genommen.

Mit der Erforschung von Archivmöbeln als Medien ist auch ein Desiderat Kittlers eingelöst, für den diese „Maschinenparts ja fast Agenten [der] Bildungsgeschichte“ (Kittler 2000, 113) sind.

Die Archivbox als analoges ,Aufbewahrungssystem‘ schreibt zwar nicht, vielmehr wird sie von Archivaren beschriftet und ist als Medium augenscheinlich träge und passiv, hat aber als Verpackung eine funktionelle Bedeutung. 
Im Unterschied zur Kunstkammer der Frühen Neuzeit, deren „Möbel die verschiedensten Objekte kunstvoll und geschickt verschachteln sollten“ (Hackenschmidt und Engelhorn 2001, 176), geht es bei der Aufbewahrung von Archivalien im modernen Literaturarchiv nicht um ein Moment der Überraschung durch ein unvorhersehbares Kombinieren der enthaltenen Dokumente (oder Objekte), sondern um die rasche Wiederauffindbarkeit und adäquate Lagerung in konservatorischer Hinsicht. Im Idealfall ist ein Nachlassbestand derart nach den RNA geordnet und indiziert, sodass sich potenzielle Archivbenutzer mittels des Bestandsverzeichnisses sofort einen Überblick darüber verschaffen können, was sich in den jeweiligen Archivboxen befindet.

Schriftgutbehälter und Archivgutbehälter haben in diesem Kontext eine dienende Funktion: Sie sollen die geordnete und geschützte Aufbewahrung des Schriftgutes ermöglichen, denn bis ins 18. Jahrhundert waren Akten noch als „lose aufeinander gestapelte Schriftstücke oder als ,Büschel“ (Faszikel)“ gefaltet und verschnürt, die „fast unverwüstlich“ (Leesch 2017) in „mächtigen Regalen tiefengestapelt, mit den nach vorne zeigenden, registraturmäßig beschrifteten Deckpappen stehend aufbewahrt“ (Hochedlinger 2009, 44) wurden. Schriftgut kann jedoch auch durch unzweckmäßige Lagerung gefährdet werden, wie z. B. infolge der Büroreform des 20. Jahrhunderts ersichtlich wurde. Johannes Papritz, der in den 1950er/60er-Jahren die Archivschule Marburg leitete, konstatiert als „folgenschwersten Eingriff der Technik in die Aufbewahrungsmöglichkeiten des Schriftgutes“ (Papritz 1957, 81) die Verwendung des Stehordners und Schnellhefters, die nicht registraturfähig seien und ob ihrer invasiven Methode - man denke an das Stanzen der Löcher - wenn schon nicht für die Aufbewahrung von Akten, so schon gar nicht für die Archivierung von Literatur geeignet sind.

Bereits Johann Heinrich Franz von Löher, der ab 1909 im Rang eines Reichsarchivrates stand, führte 1876 in der Archivalischen Zeitschrift aus, dass die Aufstellung von Archivalien so erfolgen sollte, dass ,jedes Stück möglichst geschont werde, und dass ein jedes sich leicht und handlich aus seiner Reihe herausnehmen und ebenso in dieselbe wieder hineinstellen lasse, gleichwie ein Buch in einer Bibliothek“ (Löher 1876, 64-65).

Trotz der gemeinsamen materiellen Konvergenzen haben Bücher, Akten und Handschriften eben einen anderen Status hinsichtlich ihrer Archivierung. Ernst hat festgestellt, dass es einen Unterschied macht, ob „eine Druckschrift Teil eines Archiv-Moleküls ist, also eines Faszikels oder eines Kartons, im Unterschied zur Einreihung in die Ordnung der Bibliothek“ (Ernst 2003, 44). Es sind Medien, die sich im Archiv nicht vermischen dürfen, obwohl dies - worauf etwa Johannes Sass (1924, 463-471) hinweist - oft genug vorzukommen schien. 


\section{Historische Formation(en) der Archivbox}

In einem Interview äußert sich der österreichische Schriftsteller Gerhard Roth bezüglich seiner Selbstarchivierungspraxis:

Am Anfang war sehr wenig in Ordnung. Es war eine alte Biedermeierkommode mit drei breiten Laden, in die ich einfach alles hineingegeben hab. Dann hab ich angefangen zu trennen, die Fotografien und die Notizbücher und die Manuskripte. Dann ist die Kommode zu klein geworden, und ich hab ein Zimmer genommen und die hervorragenden Bananenschachteln instrumentalisiert. - Die Bananenschachteln sind deshalb hervorragend, weil man sie aufheben kann und viel Platz hat. (Roth 2002, 101)

Roths Ausführungen zeigen aufschlussreiche Parallelen zur historischen Entwicklung und Herausbildung der Archivierungstechnik und -praxis: Seit dem Mittelalter dienten Schränke dem Schutz, aber auch der Präsentation des Sammlungsgutes. In diesem Kontext ist die Truhe, als „Universalmöbel des Mittelalters“ (Heesen 2007, 91), ein zentraler Ordnungsraum und ein der Epoche immanentes ,Aufbewahrungssystem', bis im Laufe des 17. und 18. Jahrhunderts neben dem Kabinettschrank das ,Repositorium` aufkam, ein unscharfer Begriff, der sowohl Schränke, Regale als auch Abstelltische bezeichnete.

Vielleicht ist es nur folgerichtig, dass das Medium Handschrift eben aus jenem Medium in die Archivbox zurückkehrt, mit dem es - jedenfalls in materieller Hinsicht - verwandt ist: dem Buch. Tatsächlich führte der Buchdruck durch die selektive oder kontingente Überführung von Handschriften in Buchdruck (und die damit einhergehende sukzessive Zerstörung handschriftlicher Druckvorlagen) zu einem Traditionsverlust (vgl. Müller 1988, 203-217). Die Archivbox ist als ein intermittierendes Medium zu betrachten, das diesen Traditionsverlust zu minimieren versucht, so lange natürlich die Überlieferungsbildung gegeben ist.

Dieser Konfiguration der Aufbewahrung von Schriftgut ist zunächst ein anderer Behälter vorzuziehen, nämlich das ,Buch` selbst, denn es ist ja materialiter „eine Sammlung mit einem Behälter. Dieser besteht äußerlich aus zwei Deckeln und einem Rücken. Sein Inneres ist gefüllt mit Blättern. Indem der Umschlag diesen Inhalt umschließt, gewährt er ihm einerseits Schutz, andererseits Zusammenhalt“ (Sommer 2002, 228). Während (die senkrecht aufgestellten) Bücher einer Bibliothek sicher in einem festen Einband aus Pappe ,stecken' und (zumeist) in mehreren Exemplaren vorliegen, besitzen Archivalien den Status der Einzigartigkeit, sind dementsprechend schutzbedürftig: „Bücher stecken gesichert in festem Einband: Akten und Urkunden liegen offen. Von Büchern giebt es gewöhnlich mehrere Exemplare: von Akten und Urkunden nur das eine im Archiv“ (Löher 1890, 285). 
Ein spezifischerer Vorläufer des ,Aufbewahrungssystems‘ Archivbox zeigt exemplarisch die Verschränkung der beiden Medien ,Buch` und ,Schachtel' und tritt in der zweiten Hälfte des 18. Jahrhunderts auf. In ihrer Medialität und Produziertheit sind Buch und Schachtel zunächst untrennbar miteinander verbunden, wobei ein Grund darin liegt, dass ein einzelnes Blatt „liegen, fallen, vielleicht ein bißchen schweben“ könne, so Walter Seitter, aber eben nicht „stehen kann“ wie ein Buch in einer Bibliothek (Seitter 2002, 201).

Das zeigt sich nicht nur hinsichtlich Aspekten der Kodikologie, deren Hilfswissenschaft, die ,Einbandkunde‘, Grundtypen des mittelalterlichen Bucheinbandes identifiziert hat: Codices mit Holzdeckeln, Hüllenbuch, Beutelbuch, Koperteinband und Aktenband (vgl. Kümper 2014, 161). Die Beziehung dieser beiden ,Medien“ ist auch deswegen aufschlussreich, wie Heinz Schmidt-Bachem in seiner materialreichen und ausführlichen Darstellung der Kultur- und Wirtschaftsgeschichte der Papier verarbeitenden Industrie festgestellt hat, da die Wurzeln der KartonagenIndustrie, die bislang wenig bekannt und nicht systematisch erforscht sind, im Buchbinderhandwerk liegen (vgl. Schmidt-Bachem 2011, 573).

Besieht man sich eines der ersten Beispiele für die Schachtel als ,Aufbewahrungssystem', kommt auch bibliothekarisches Know-how hinzu: Daniel Charles Solander, Botaniker, Weltumsegler und Schüler des Naturhistorikers Carl von Linné, hatte in seiner Funktion als Archivar und Bibliothekar von 1773 bis 1782 an der British Library in London eine (allerdings aus weichem Holz gefertigte) Schachtel konstruiert, die, einem kunstvoll aufklappbaren Buch ähnelnd, Platz für Dokumente oder auch naturwissenschaftliche Objekte bot. 1844 wird dieses ,Aufbewahrungssystem“ folgendermaßen beschrieben: „a wooden box, backed with leather, the sides covered with marble or other paper, having leather corners, so that it has much the appearance of a book; one of the sides is made to open as a lid, carrying with it the back which is attached to it [...]“ (zit. n. Caladaro 1993, 388).

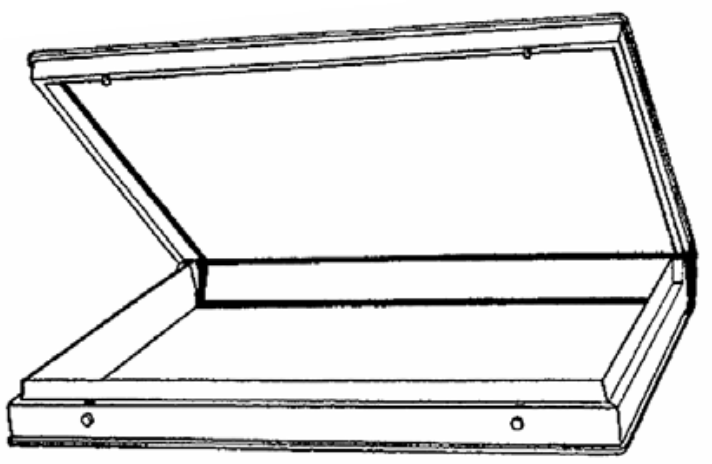

Abb. 2: Solander-Box. 
Johann Wolfgang von Goethe fertigte große Briefumschläge, die er „Säcke“ oder „Kapseln“ nannte, an, in denen er seine Papiere und „Acten“ zu verwahren pflegte, bevor 1822 sein Nachlass durch den Weimarer Hofbibliothekar Friedrich Theodor Kräuter geordnet wurde. Ernst Robert Curtius hat in einem Essay zu Goethes Aktenführung darauf hingewiesen, dass er die großen Briefumschläge, die er zur Verwahrung seiner Papiere zu verwenden pflegte, nicht nur als „Tasche“, sondern auch als „Sack“ bezeichnete. In diesem Zusammenhang verwendet er u. a. den Begriff „Tecturen“, dies bezeichnet - so vermutet Curtius - Aktendeckel, also den Umschlag eines Aktenstücks (vgl. Curtius 1999, 164). Die Umhüllung hat hier noch eine zweidimensionale Qualität. Wie Carlos Spoerhase festgestellt hat, äußert sich Goethe in verschiedenen Korrespondenzen oft über leere, beschriebene und bedruckte Papierbogen, die sowohl als materielle Träger von Briefen oder Drucken, aber eben auch als Verpackungsmaterial thematisiert werden (vgl. Spoerhase 2018, 588). Erst die Faltung mit ihren gekrümmten und beweglichen Grenzflächen, die „ein oben und unten, ein innen und außen in nur temporär entscheidbarem Zustand“ (Zinsmeister 2004, 164) beschreibt, markiert den Übergang einer zweidimensionalen Fläche in ein dreidimensionales Gebilde. Während Goethe bei seinen Anordnungen in der zweiten Dimension verblieb und noch nicht den Schritt vom Faszikel zur Archivschachtel vollzog, verhält es sich mit den Handschriftensammlern des 19. Jahrhunderts anders.

Jene „Verwandte[n] der Archivare wie der Philologie“ (Müller 2012, 289) sind als Rivalen der noch nicht institutionalisierten Gruppe der Literaturarchivare diesen um einige konservatorische Schritte voraus, behandelt doch der Archivar und Schriftsteller Ludwig Bechstein in seinen Ausführungen über die Autographensammlung für Die Wissenschaften im neunzehnten Jahrhundert (1858) sehr genau das Handbuch für Autographensammler von Johann Günther und Otto August Schulz, das aufgrund seiner Systematisierung und praktischen Hinweise zur Anlage einer Sammlung als bedeutender „,Markstein“ in der Entwicklung des Autographensammelns“ (Plachta 2010, 84) gilt.

Der fünfte Abschnitt des Handbuchs führt in das „Labyrinth der SammlerSysteme“ (Bechstein 1858, 232) und zeigt als Sammlungs- und Aufbewahrungssystem für wertvolle Handschriften einen „Autographen-Carton“ in Buchform:

Die Grundlage dieses Cartons bildet ein aus verhältnissmässig schwachem weichen Holze gearbeiteter 4-5 Zoll hoher Rahmen, welcher an der rechten Seite, gleich einem Buche, mit einer Hohlkehle versehen wird. Diesen nimmt der Buchbinder in die Arbeit und giebt ihm durch zwei Deckel, von denen der untere befestiget, der obere aber mittelst eines angebrachten Charniers zum Auf- und Zumachen eingerichtet ist, und durch einen Rücken die Buchform. (Günther und Schulz 1856, 138) 


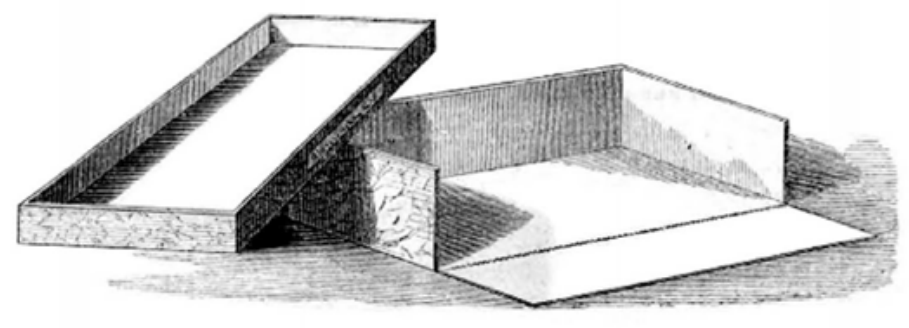

Abb. 3: „Autographen-Carton“.

Das Aufkommen dieser „Autographen-Cartons“ und die sukzessiv erfolgende diskursive Ordnung des modernen Literaturarchivs verdanken sich im 19. Jahrhundert auch Innovationsschüben der papierverarbeitenden Industrie, welche die Verwendung von (Falt-)Schachteln für Zwecke der Archivierung begünstigte. In der ersten Hälfte des 19. Jahrhunderts wuchs die „Zahl der papierenen Objekte in der Dingwelt weit über die Welt der Bücher, Zeitungen und Briefe, der Formulare und Akten“ (Müller 2012, 297-298) hinaus. Die massenhafte Produktion von Kartonagen, Tüten und Taschen - begünstigt durch die Einführung der Rundsiebpapiermaschine (Pappenmaschine) im Jahr 1830 -, die als Verpackungsmaterial Verwendung fanden, ließen Pappe und Karton zirkulieren. In der Buchbinderei fanden vor allem graue Pappen Verwendung, die aus Lumpen und Papierabfällen hergestellt wurden. Diese zeichneten sich durch ihre besondere Zähigkeit und Biegsamkeit aus und taugten daher besonders für Bucheinbände (vgl. Biesalski 1991, 63). 1890 erfand der Engländer Henry M. Smith die Schachteln aus (doppelseitiger) Wellpappe, er entwickelte die sogenannte Durchzug- oder Gürtelschachtel (vgl. Schmidt-Bachem 2011, 579); dieser Typ wurde erst später durch die Faltschachtel aus Wellpappe ersetzt. Hinsichtlich der maschinellen Fertigung waren die Gebrüder Brehmer mit der Entwicklung der Fadenheftmaschine zentral, das weiterentwickelte Verfahren der Drahtheftmaschine und später der Faden-Buchheftmaschine revolutionierte sowohl die Buchherstellung, ähnlich wie die Einführung der Schnellpresse im Druckbereich, als auch die Herstellung von Schachteln (vgl. Schmidt-Bachem 2011, 587).

1894 sprach der österreichische Germanist Jakob Minor eher abfällig davon, dass „Centralanstalten für litteraturgeschichtliche Hilfsarbeiten“, eben diejenigen Institutionen, die Wilhelm Dilthey (1970 [1899], 2-16) in seinem Vortrag als „Archive für Literatur“ bezeichnet und gefordert hatte, keine bloßen „Stapelplätze“ sein sollten, wo „Papiere in Empfang“(Minor 1894, 2) genommen werden. Vielmehr entwirft der archivaffine Minor, er hatte u. a. auch an der „Sophienausgabe“ der Werke Goethes mitgearbeitet, aus der Perspektive des Literaturprofes- 
sors - zu dieser Zeit war er Ordinarius an der Universität Wien - einen sehr bestimmenden Arbeitsauftrag für Literaturarchivare. Vielleicht hat Minor auch im Krünitz nachgeschlagen, denn dieser wusste unter dem Lemma Staatsarchiv darüber Bescheid, dass „[i]n einigen Archiven [...] die Urkunden auch in Pappschachteln oder Kasten verwahrt [werden], welche in Bücherrepositorien gestellt werden, das heißt, die Repositorien haben dieselbe Einrichtung und Gestalt der Bücherrepositorien, und darein werden die Schachteln oder Kisten eine auf die andere gesetzt [Hervorhebung, S. M.]“ (Krünitz 1836, 160). Auch für Joseph Alexander Freiherr von Helfert sind in seinem Staatlichen Archivwesen dreierlei Aufbewahrungssysteme intelligibel, nämlich „Actenbündel[], Registerbände[] und Pappschachteln“ (Helfert 1893, 10).

Dass Minor den hier eher pejorativen Begriff „Stapelplätze“ benutzt, ist mediengeschichtlich aufschlussreich, spricht man den ,Verpackungen“, die „keine bloßen Mittel“, aber auch „keine ganz unterwürfigen Sklaven sind“, so Walter Seitter (2002, 202), eine eigene Konfiguration zu. Dilthey spricht in seinem Gründungsmanifest des Literaturarchivs von hilflosen Papiermassen und davon, dass Nachlass-Erben die Papiermassen zunächst „noch sorgsam in einem Schrank“ aufbewahren, aber diese schließlich „meist in einer Kiste auf dem Speicher“ (Dilthey 1970, 9) landen würden. Zwar antizipiert er in seinem Aufruf die Sicherung, Zusammenführung, systematische Anordnung und Erschließung der Handschriften deutscher Autoren seit dem Humanismus, aber welches Dispositiv der Aufbewahrung und konservatorischen Praxis zum Tragen kommt, thematisiert er nicht. Dieses wird jedoch ersichtlich, konsultiert man die Festschrift der Litteraturarchiv-Gesellschaft zu ihrem 25-jährigen Bestehen:

Durch ein Übereinkommen mit der k[öni]gl[ichen]. Bibliothek in Berlin erhielten wir in den Räumen derselben Platz zum Aufstellen von Schränken, in denen unsere Sammlungen untergebracht sind. Die gebundenen Handschriften und die in Pappkartons befindlichen Konvolute von losen Blättern sind unter den Namen der Verfasser zusammengestellt; [...]. (Meisner 1916, 13-14)

In der Kodifizierung der europäischen Archivtheorie, der sogenannten „Dutch Manual“ von 1898, die 1905 als Anleitung zum Ordnen und Beschreiben von Archiven in deutscher Übersetzung erschien, wird hinsichtlich der wichtigsten Prinzipien der Aufstellung und Anordnung des Archivinventars artikuliert, dass die „Bewahrung der [Archiv-]Stücke ganz frei“ zu entscheiden sei, aber die „Sorge für ihre gute Erhaltung“ (Mueller et al. 1905, 94) gegeben sein müsse. Hier ist die Archivbox noch ein passives Medium, das den Platz eines Aktenstückes innerhalb der Gesamtstruktur des Archivs bezeichnet und über den Umfang informieren soll (vgl. Mueller et al. 1905, 96 u. 120). 


\section{Schluss}

Die Archivbox, mittlerweile als Medium ausdifferenziert und von Design sowie Konstruktion kodifizierten Prozeduren unterworfen, ist zwar ein geeignetes ,Aufbewahrungsystem;, das den Nachlass vor äußeren Einflüssen schützt, diesen jedoch auch gleichsam in seiner Vielgestaltigkeit bezwingt und in eine spezifische konservatorische Figuration zwingt. Als Grundlage für die Eignung von Schachteln im archivarischen Kontext ist mittlerweile ihre Alterungsbeständigkeit, Materialzusammensetzung und mechanische Festigkeit über DIN-Normen geregelt (vgl. Korn 2006, 93). Die beste Art der Lagerung von Archivalien im Literaturarchiv besteht deshalb darin, diese in festen, geschlossenen Boxen aus Karton aufzubewahren: „Verwenden Sie zur Lagerung von Werken aus Papier, Büchern und Archivalien Produkte, welche der Norm ISO 9706 für alterungsbeständiges Papier bzw. ISO 16245 für Archivschachteln und Archivhüllen entsprechen“ (Huber und Lerber 2015, 109). Es bedürfte wohl noch genauerer und konziserer Studien, um dieses ,Aufbewahrungssystem', seine Entwicklung, Ausdifferenzierung und Anwendungsformen weiter in einen Kontext der Wissensordnungen und der normativen Praktiken des Archivs zu stellen.

Oftmals wird - ganz im Sinne des eingangs zitierten Roland Barthes - der Charakter der Schachtel als ein Emballagegegenstand vergessen, der seiner Natur nach dem Käufer in Rechnung gestellt wird. Wie Klaus Kastberger angemerkt hat, bedarf es ja eines „nicht unerheblichen materiellen Einsatzes“, um die „Kartons und Papierstapel in Nachlässe zu verwandeln, also wirklich ins Archiv aufzunehmen“ (Kastberger 2017, 427). Zudem stellt er die Frage, ob der Nachlass vielleicht nur deshalb aus „Werk- und Werk-Außerhalb“ zusammengesetzt ist, um ihn bequem im ,Aufbewahrungssystem‘ Archivbox ablegen zu können. Dem Bestand - so kritisiert auch Ulrich Raulff - werden durch einen „Vorgang der Neutralisierung“ seine Attribute entzogen, dabei spielt auch die Archivbox eine wesentliche Rolle:

Durch die an eine militärische Investitur erinnernde Einlagerung der Papiere in Mappen einheitlicher Größe und Farbe (grau) und Kästen von gleicher Größe (die geeignet sind, einen Maßstab zur Quantifizierung von Archivgut abzugeben) wird eine reine Quantität geschaffen. Die wilde und bedrohliche Singularität der Nachlässe wird in der Uniformität des Archivguts gebändigt. (Raulff 2009, 229) 


\section{Literaturverzeichnis}

Barthes, Roland: Das Reich der Zeichen. Frankfurt a. M.: Suhrkamp, 2012.

Bechstein, Ludwig. „Die Autographensammlung“. Die Wissenschaften im neunzehnten

Jahrhundert. Ihr Standpunkt und die Resultate ihrer Forschungen. Bd. 3. Hg. J. A. Romberg. Leipzig, 1858. 215-236.

Benne, Christian: Die Erfindung des Manuskripts. Berlin: Suhrkamp, 2015.

Biesalski, Ernst-Peter: Die Mechanisierung der deutschen Buchbinderei 1850-1900. Frankfurt a. M.: Buchhändler-Vereinigung, 1991.

Bredekamp, Horst: Antikensehnsucht und Maschinenglauben. Die Geschichte der Kunstkammer und die Zukunft der Kunstgeschichte. Berlin: Wagenbach, 1993.

Brenneke, Adolf: Archivkunde. Ein Beitrag zur Theorie und Geschichte des europäischen Archivwesens. Bearb. n. Vorlesungsnachschriften und Nachlaßpapieren und erg. v. Wolfgang Leesch. Leipzig: Koehler \& Amelang, 1953.

Caladaro, Nicolai. „The solander box: Its varieties and its role as an archival unit of storage for prints and drawings in a museum, archive or gallery“. Museum Management and Curatorship 12.4 (1993): 387-400.

Curtius, Ernst Robert. „Goethes Aktenführung“. Grundlagen der Literaturwissenschaft. Exemplarische Texte. Hg. Bernhard J. Dotzler. Köln, Weimar, Wien: Böhlau, 1999. 158-167.

Dilthey, Wilhelm. „Archive für Literatur“. Gesammelte Schriften. Bd. 15: Zur Geistesgeschichte des 19. Jahrhunderts. Porträts und biographische Skizzen, Quellenstudien und Literaturberichte zur Theologie und Philosophie im 19. Jahrhundert. Hg. Karlfried Gründer. Göttingen: Vandenhoeck \& Rupprecht, 1970. 2-16.

Ebeling, Knut, und Stephan Günzel. „Einleitung“. Archivologie. Theorien des Archivs in Wissenschaft, Medien und Künsten. Hg. Knut Ebeling und Stephan Günzel. Berlin: Kadmos, 2009. 7-28.

Ernst, Wolfgang. Im Namen von Geschichte. Sammeln - Speichern - Er/zählen. Infrastrukturelle Konfiguration des deutschen Gedächtnisses. München: Fink, 2003.

Farge, Arlette. Der Geschmack des Archivs. Göttingen: Wallstein, 2011.

Friedrich, Markus. Die Geburt des Archivs. Eine Wissensgeschichte. München: Verlag Oldenbourg, 2013.

Gfrereis, Heike, und Ellen Strittmatter (Hg.): Zettelkästen. Maschinen der Phantasie. Marbach a. Neckar: Deutsche Schillergesellschaft, 2013.

Glauert, Mario. „Verpackungen für Archivgut. Empfehlungen der Archivreferentenkonferenz, ausgearbeitet vom Bestandserhaltungsausschuss der ARK“. Der Archivar 64.1 (2011): 57-62.

Grimm, Jacob, und Wilhelm. Deutsches Wörterbuch. 16 Bde. In 32 Teilbänden. Leipzig: Hirzel, 1854ff. http://woerterbuchnetz.de (30.10.2018).

Hackenschmidt, Sebastian, und Klaus Engelhorn (Hg.). Möbel als Medien. Beiträge zu einer Kulturgeschichte der Dinge. Bielefeld: transcript, 2011.

Hall, Murray G., und Gerhard Renner: Handbuch der Nachlässe und Sammlungen österreichischer Autoren. Wien, Köln, Weimar: Böhlau, 1992.

Hammer-Luza, Elke. „Von der Urkundenlade zur modernen Archivbox. Lagerungstechniken im Laufe der Jahrhunderte am Beispiel der Steiermark“. Die Kunst des Archivierens. Hg. Josef Riegler. Graz: Steiermärkisches Landesarchiv, 2007. 57-71. 
Heesen, Anke te, und E. C. Spary. „Sammeln als Wissen“. Sammeln als Wissen. Das Sammeln und seine wissenschaftsgeschichtliche Bedeutung. Hg. Anke te Heesen und E. C. Spary. Göttingen: Wallstein, 2001. 7-21.

Heesen, Anke te. Der Zeitungsausschnitt. Ein Papierobjekt der Moderne. Frankfurt a. M.: Fischer, 2006.

Heesen, Anke te. „Vom Einräumen der Erkenntnis“. Auf \Zu. Der Schrank in den Wissenschaften. Hg. Anke te Heesen und Anette Michels. Berlin: Akademie Verlag, 2007.

Henningsen, Thorvald. Das Handbuch für den Buchbinder. 2. Aufl. St. Gallen: Hostettler, 1969. Huber, Joachim, und Karin von Lerber. Handhabung und Lagerung von mobilem Kulturgut. Ein Handbuch für Museen, kirchliche Institutionen, Sammler und Archive. Bielefeld: transcript, 2015.

Kastberger, Klaus. „Nachlassbewusstsein. Vorlass-Chaos und die Gesetze des Archivs. Am Beispiel Friederike Mayröckers“. In: Nachlassbewusstsein. Literatur, Archiv, Philologie 1750-2000. Hg. Kai Sina und Carlos Spoerhase. Göttingen: Wallstein, 2017. 409-428.

Korn, Henriette. „Archivboxen im Test. Zur Aufbewahrung von Urkunden und Siegeln“. Arbeitsblätter des Arbeitskreises Nordrhein-Westfälischer Papierrestauratoren 10 (2006): 93-99.

Krawjeski, Markus. ZettelWirtschaft. Die Geburt der Kartei aus dem Geiste der Bibliothek. Berlin: Kadmos, 2017.

Kramer, Anke, und Annegret Pelz (Hg.). Album. Organisationsform narrativer Kohärenz. Göttingen: Wallstein, 2013.

Krünitz, Johann Georg. Oekonomische Encyklopädie, oder allgemeines System der Staats-, Stadt, Haus- $u$. Landwirthschaft. Bd. 138: Säure-Schaf. Bruenn: Traßler, 1824. http://www. kruenitz1.uni-trier.de (30.10.2018).

Krünitz, Johann Georg. Oekonomische Encyklopädie, oder allgemeines System der Staats-, Stadt, Haus- $u$. Landwirthschaft. Bd. 164: Staat, (Hof-) - Staatsschrift. Bruenn: Traßler, 1836. http://www.kruenitz1.uni-trier.de (30.10.2018).

Kümper, Hiram. Materialwissenschaft Mediävistik. Eine Einführung in die historischen Hilfswissenschaften. Paderborn: Schöningh, 2014.

Leesch, Wolfgang. „Schriftgutbehälter“. Lexikon des gesamten Buchwesens Online. 2017. http://dx-doi-org.uaccess.univie.ac.at/10.1163/9789004337862_COM_190762 (30.10.2018).

Löher, Franz von. Archivlehre. Grundzüge der Geschichte, Aufgabe und Einrichtung unserer Archive. Paderborn: Schöningh, 1890.

Meisner, Heinrich. Die Litteraturarchiv-Gesellschaft während der fünfundzwanzig Jahre ihres Bestehens 1891-1916. Ein Überblick. Berlin: Litteraturarchiv-Gesellschaft, 1916.

Minor, Jakob. „Centralanstalten für litteraturgeschichtliche Hilfsarbeiten“. Euphorion 1.1 (1894): 17-26.

Mueller, Samuel, J. A. Feith, und Robert Fruin. Anleitung zum Ordnen und Beschreiben von Archiven. Leipzig: Harrassowitz, 1905 [1898].

Müller, Jan-Dirk. „Der Körper des Buches. Zum Medienwechsel zwischen Handschrift und Druck“. Materialität der Kommunikation. Hg. Hans Ulrich Gumbrecht und Ludwig K. Pfeiffer. Frankfurt a. M.: Suhrkamp, 1995. 203-217.

Müller, Lothar. Weiße Magie. Die Epoche des Papiers. München: Hanser, 2012.

Papritz, Johannes. „Organisationsformen der Schriftgutbewahrung in der öffentlichen Verwaltung. Historische Grundlagen und Stand der Probleme“. Der Archivar 10 (1957): 275-294. 
Plachta, Bodo. „Werkstatt, Archiv, Pantheon“. Die Werkstatt des Dichters. Imaginationsräume literarischer Produktion. Hg. Klaus Kastberger und Stefan Maurer. Berlin, Boston: de Gruyter, 2017. 29-45.

Poppe, Johann Heinrich Moritz von. Der Papparbeiter, Papiermachéarbeiter und Papierkünstler Oder die Kunst, aus Pappe, Papierteig und Papier allerlei nützliche schöne Sachen zu verfertigen. Ulm: Ebner, 1840.

Raulff, Ulrich. „Sie nehmen gern von den Lebendigen. Ökonomien des literarischen Archivs“. Archivologie. Theorien des Archivs in Wissenschaft, Medien und Künsten. Hg. Knut Ebeling und Stephan Günzel. Berlin: Kadmos, 2009. 223-232.

Roth, Gerhard. „Die hervorragenden Bananenschachteln. Gespräch mit Wendelin SchmidtDengler und Lucas Cejpek im Österreichischen Literaturarchiv der Österreichischen Nationalbibliothek, Wien 30.11.2000“. manuskripte 42.157 (2002): 101-106.

Sass, Johannes. „Bücher in Akten“. Zentralblatt für Bibliothekswesen 41 (1924): 463-471.

Schmidt-Bachem, Heinz. Aus Papier. Eine Kultur- und Wirtschaftsgeschichte der Papier verarbeitenden Industrie in Deutschland. Berlin, Boston: de Gruyter, 2011.

Schüller-Zwierlein, André. „Diachrone Zugänglichkeit: Versuch einer Prozesstypologie“. Diachrone Zugänglichkeit als Prozess. Kulturelle Überlieferung in systematischer Sicht. Hg. Michael Hollmann und André Schüller-Zwierlein. Berlin, München, Boston: de Gruyter, 2014. 15-80.

Schwab, Werner. In harten Schuhen: ein Handwerk. Hg. Ingeborg Orthofer. Graz, Wien: Droschl, 1999.

Seitter, Walter. Physik der Medien. Materialien, Apparate, Präsentierungen. Weimar: Verl. f. Geisteswissenschaften, 2002.

Sinnhold, A. Der Archivar und das Archivwesen sowie deren Verbindung mit den Kanzleiexpeditionen, als zweckmäßigste Einrichtung zur Beförderung eines geregelten, übersichtlichen und sicheren Ganges der Archivverwaltung und des Kanzleiexpeditionswesens. Weimar: Voigt, 1842.

Sommer, Manfred. Sammeln. Ein philosophischer Versuch. Frankfurt a. M.: Suhrkamp, 2002. Spoerhase, Carlos, Dirk Werle und Markus Wild. „Unsicheres Wissen. Zur Einführung“. Unsicheres Wissen. Skeptizismus und Wahrscheinlichkeit, 1550-1850. Hg. Carlos Spoerhase, Dirk Werle und Markus Wild. Berlin, New York: de Gruyter, 2009. 1-13. Spoerhase, Carlos. Das Format der Literatur: Praktiken materieller Textualität zwischen 1740 und 1830. Göttingen: Wallstein, 2018.

Vismann, Cornelia. Akten. Medientechnik und Recht. Frankfurt a. M.: Fischer, 2000.

Wolf, Burkhardt. „Räume des Wissens“. In: Handbuch Literatur \& Raum. Hg. Jörg Dünne und Andreas Mahler. Berlin, Boston: de Gruyter, 2015. 115-124.

Zinsmeister, Annett. „Transformation und Faltung“. Digitale Transformationen. Medienkunst als Schnittstelle von Kunst, Wissenschaft, Wirtschaft und Gesellschaft. Hg. Monika Fleischmann und Ulrike Reinhard. Heidelberg: whois verlag- und vertriebsgesellschaft, 2004. 164-170. 